\title{
Design of 3.7GHz BeO Ceramic Window
}

\author{
Yunying Tang, Fukun Liu, Xiaojie Wang \\ Institute of Plasma Physics, Chinese Academy of \\ Sciences, Hefei, China \\ yytang@ipp.ac.cn, fkliu@ipp.ac.cn, xjiew@ipp.ac.cn
}

\begin{abstract}
The design of $3.7 \mathrm{GHz}$ ceramic window is needed in the Lower Hybrid Current Drive (LHCD) system of Experimental Advanced Superconducting Tokamak (EAST). The material of ceramic disk chooses $\mathrm{BeO}$ which owns low dielectric constant, low loss tangent and high thermal conductivity. RF results obtained from the analysis of HFSS software show that the voltage standing wave ratio (VSWR) is less than 1.05 during the bandwidth from $3.5 \mathrm{GHz}$ to $3.9 \mathrm{GHz}$. Thermal analysis is analyzed by ANSYS finite element software. When the water flow rate is $0.36 \mathrm{t} / \mathrm{h}$, the maximum temperature differential on the ceramic disk is $26^{\circ} \mathrm{C}$ and the thermal stress is in a safe range.
\end{abstract}

Keywords-BeO; Al2O3; Ceramic window; RF analysis; thermal analysis

\section{INTRODUCTION}

Ceramic window [1] is a key microwave component in the LHCD system on EAST. EAST [2] researches the steady-state and advanced operation of the plasma, which is served for the fusion energy projection. LHCD system [3] which is one of the main auxiliary heating methods in EAST experiment assists the plasma to reach to the ignition temperature. There is vacuum state in EAST vessel, nevertheless the LHCD system is aerated by nitrogen. The ceramic window is designed to separate the high vacuum of EAST vessel from atmospherically pressured transmission lines of the LHCD system.

In the traditional design of ceramic window, the separating ceramic disk chooses $\mathrm{Al}_{2} \mathrm{O}_{3}$ material [4]. $\mathrm{Al}_{2} \mathrm{O}_{3}$ material can achieve fine RF function, however it owns low thermal conductivity. In each LHCD transmission line, $250 \mathrm{~kW}$ continuous wave (CW) energy, maybe higher in the future, transmits through the ceramic window and injects into the plasma of EAST. Because of the dielectric loss of ceramic material, part of high power microwave energy will deposit on the ceramic disk. The deposited energy will be taken away by cooling water which is surrounded the ceramic disk. However, if the deposited power is too much to be taken away, the ceramic disk will crack and the EAST experiment will be broken off. In order to avoid the fracture of ceramic disk, $\mathrm{BeO}$ material [5] is a better choice due to its dielectric constant and its loss tangent. Furthermore, the thermal conductivity of $\mathrm{BeO}$ material is high and may be ten times than $\mathrm{Al}_{2} \mathrm{O}_{3}$ material.

In the paper, the requirements to the $\mathrm{BeO}$ ceramic window is introduced in the next section. And then the modeling, the RF results and the thermal analysis are present. At the last section, the conclusions are given.

\author{
Yunying Tang \\ University of Science and Technology of China, Hefei, \\ China \\ yytang@ipp.ac.cn
}

\section{REQUIREMENTS}

LHCD system requires a wide RF bandwidth to pass high power energy on CW operation condition. The working frequency of the ceramic window is $3.7 \mathrm{GHz}$. The VSWR should be less than 1.1 at the bandwidth from $3.5 \mathrm{GHz}$ to $3.9 \mathrm{GHz}$. The ceramic window will be connected with WR284 rectangular waveguide whose size is $72.14 \mathrm{~mm} \times 34.04 \mathrm{~mm}$. The width of the ceramic window, the thickness of $\mathrm{BeO}$ ceramic disk and other geometry variables should be used to develop a design which meets the broadband VSWR requirements, enables a strong attachment, withstands more than 2 atmospheres and bears less than $10^{-}$ ${ }^{9} \mathrm{~Pa} \cdot \mathrm{m}^{3} / \mathrm{s}$ vacuum leak rate.

\section{MODELING}

The ceramic window adopts traditional design which is composed of two rectangular waveguides and one cylindrical waveguide brazed with a ceramic disk. The diameter of the ceramic disk is equal to the diagonal length of rectangular waveguide. The VSWR is determined from the reflected power and input power at the driven port. The finite element model built in HFSS software is shown in Fig. 1.

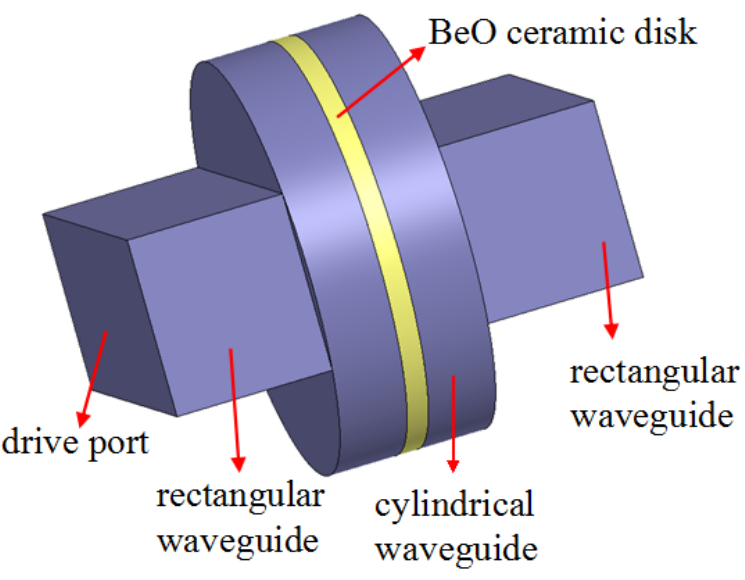

Figure 1. BeO ceramic window model 


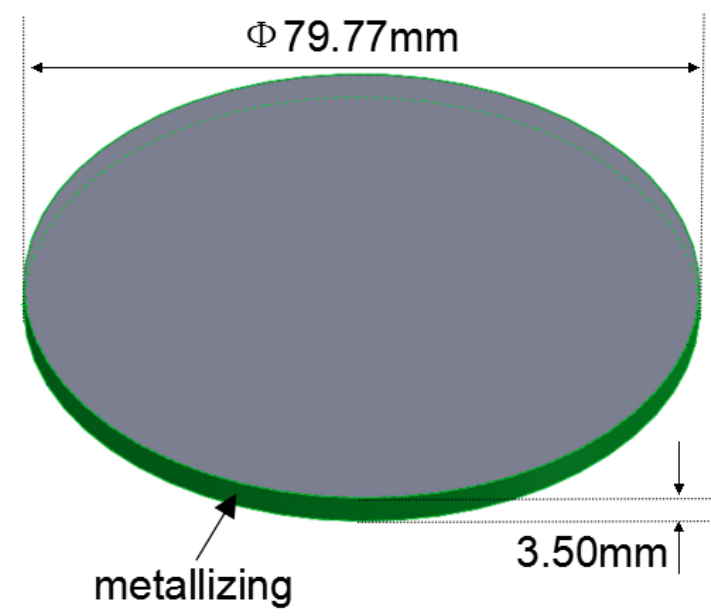

Figure 2. The parameters of $\mathrm{BeO}$ ceramic disk

From the size of rectangular waveguide, the diameter of BeO ceramic disk is $79.77 \mathrm{~mm}$. The length of the cylindrical waveguide and the thickness of ceramic disk are two key parameters which affect the RF results. After series of optimization by HFSS software, the total length of the cylindrical waveguide is $31.6 \mathrm{~mm}$ and the parameters of $\mathrm{BeO}$ ceramic disk are shown in Fig. 2. The thickness of ceramic disk is $3.5 \mathrm{~mm}$. The edge of the ceramic disk will be metallized in order to weld with the inner wall of cylindrical waveguide.

\section{RFRESULTS}

The VSWR at the range from $3.5 \mathrm{GHz}$ to $3.9 \mathrm{GHz}$ is shown in Fig. 3. At $3.7 \mathrm{GHz}$, the nominal frequency, the VSWR is determined to be 1.002. The thin thickness of $\mathrm{BeO}$ ceramic disk which can withstand more than 2 atmospheres is in favor of gaining so low VSWR at the design frequency. The VSWR within the bandwidth from $3.5 \mathrm{GHz}$ to $3.9 \mathrm{GHz}$ is below 1.05 .

The finite element code ANSYS is used for its multidiscipline capability. In the calculation of VSWR, the dielectric loss is considered. The heat loss is a function of dielectric constant, the loss tangent, the frequency and the electric field in $\mathrm{BeO}$ ceramic disk. The dielectric constant and the loss tangent of the $\mathrm{BeO}$ material are 6.9 and $8 \mathrm{e}-4$ respectively at $3.7 \mathrm{GHz}$. The passing power is $250 \mathrm{~kW}$.

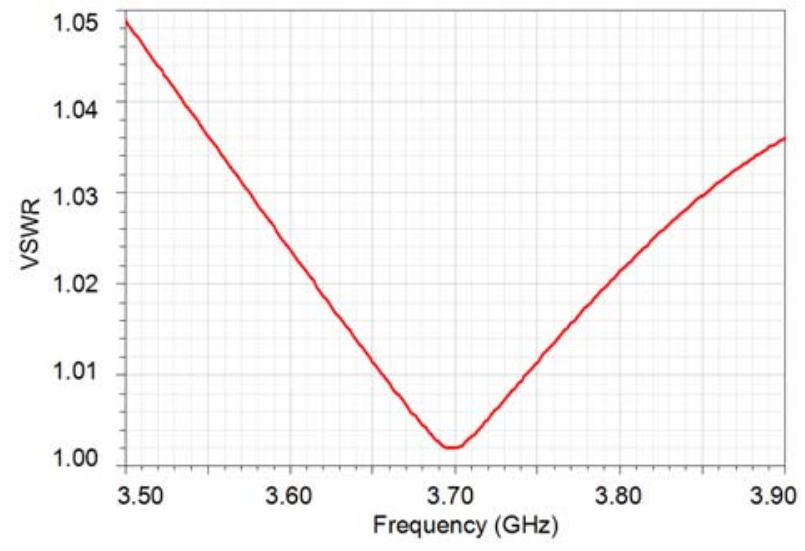

Figure 3. VSWR for required frequency range

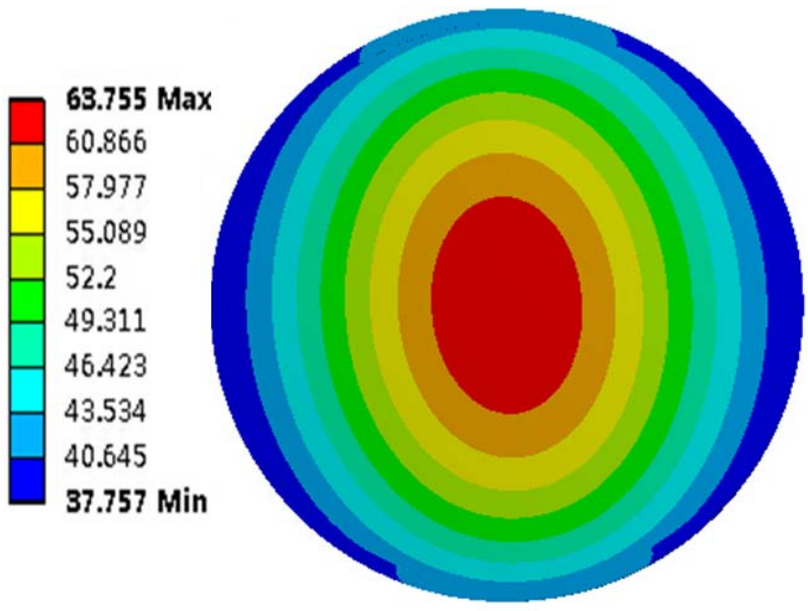

Figure 4. Ceramic window temperature contours

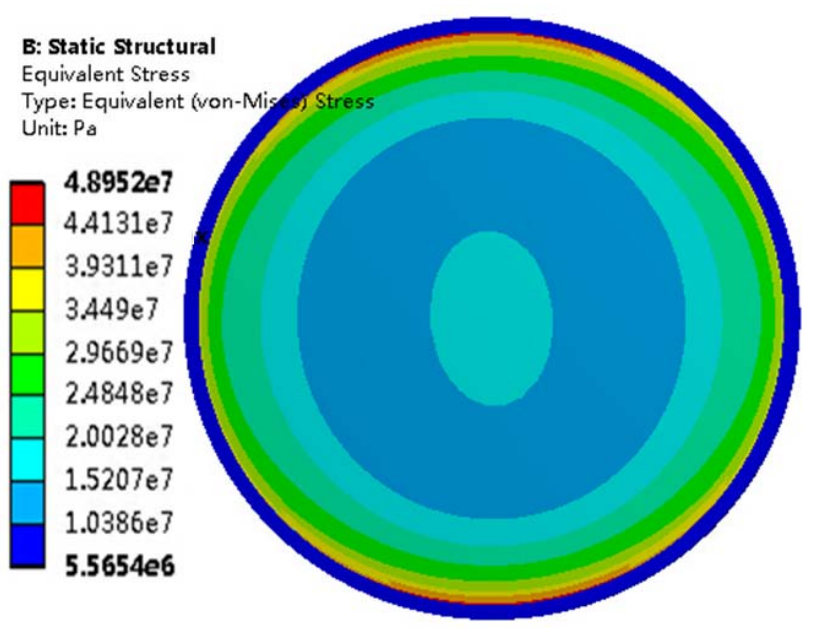

Figure 5. Thermal stress in ceramic window

\section{THERMAL ANALYSIS}

When $250 \mathrm{~kW}$ CW power passes the BeO ceramic disk, the temperature will increase. In order to decrease the 
temperature, the ceramic disk is welded with the copper wall of cylindrical waveguide inlaid the cooling channel surrounding of the ceramic disk edge. Fig. 4 shows the resulting thermal contours when the water flow rate is 0.36 $\mathrm{t} / \mathrm{h}$. The maximum temperature occurs in the center of the ceramic disk, which is the location of the maximum electric field. Owing to the high thermal conductivity of $\mathrm{BeO}$, the maximum temperature is about $64^{\circ} \mathrm{C}$ and the temperature differential is only $26^{\circ} \mathrm{C}$. The tensile strength of $\mathrm{BeO}$ is 154MPa. While in Fig. 5, the maximum stress value is 49MPa, smaller than tensile strength. Thus, thermal stress will not damage ceramic disk.

\section{CONCLUSIONS}

By the optimization of all geometry parameters, the requirements of the broadband VSWR among the bandwidth and low VSWR on working frequency are achieved. Keeping these parameters at reasonable dimensions provides for up to 2 atmospheres of pressure differential. The high thermal conductivity of $\mathrm{BeO}$ enables efficient heat removal from the window and therefore high power $>250 \mathrm{~kW}$ can pass through the ceramic window on CW operation.

\section{REFERENCES}

[1] Yun Ying Tang, Xiao Jie Wang, Liang Liu, Lian Min Zhao, Fu Kun Liu, "Design of High Power Microwave Ceramic Windows in CBan,” Journal of Fusion Energy, Vol. 33, Iss. 1, 2014, pp. 68-73.

[2] WAN Yuanxi, Li Jiangang, WENG Peide, EAST team, "First Engineering Commissioning of EAST Tokamak," Plasma Science \& Technology, Vo1. 8, No. 3, 2006, pp. 253-254.

[3] ZHAO Lianmin, SHAN Jiafang, LIU Fukun, JIA Hua, WANG Mao, LIU Liang, et al., "A 2450MHz/2MW Lower Hybrid Current Drive System For EAST,” Nuclear Electronics \& Detection Technology, Vol. 29, No. 5, Sep. 2009, pp. 1143-1148.

[4] SHI Ming, SHANG A-man, CHENG Jian, "Simulation of Al2O3 Ceramic in Millimeter Wave Window and Evaluation of Sealed Residual Stress,” Vacuum Electronics, Iss. 6, 2010, pp. 44-47.

[5] LI Xiao-ying, "Study of the Standardization of Beryllia Ceramic Materials and Products,” Vacuum Electronics, Iss. 1, 2008, pp. 58-62. 